\section{Contribución de la Red Iberoamericana de Medio Ambiente a la formación ambiental en América Latina y el Caribe.}

\section{Contribution of the Ibero American Environment Network to environmental training in Latin America and the Caribbean.}

\section{Contribuição da Rede Ibero-americana de Maio Ambiente à formação ambiental na América Latina e o Caribe.}

\author{
Yordanis Gerardo Puerta de Arma ${ }^{1}$, Sara Yaima \\ Ulloa Bonilla y Caridad Dailyn López Cruz \\ ${ }^{1}$ Red Iberoamericana de Medio Ambiente, Ecuador \\ contacto@reima-ec.org
}

Recibido:17/02/2020

Aceptado: $28 / 04 / 2020$

Publicado: 30/06/2020

\title{
RESUMEN
}

El objetivo de este trabajo fue sistematizar las experiencias de la Red Iberoamericana de Medio Ambiente (REIMA, A.C.) en la formación ambiental de líderes comunitarios para la gestión de proyectos de desarrollo endógeno, con la participación activa de grupos vulnerables y población joven en América Latina y el Caribe. La investigación demostró que la REIMA, A.C. contribuyó a dichos propósitos facilitando el intercambio de experiencias, la realización de proyectos de investigación y desarrollo, y la organización de eventos académicos relacionados con la sustentabilidad en Iberoamérica; ello como resultado de la articulación entre las más de 100 instituciones que forman parte de la misma en 17 países del orbe. La amplia participación de docentes, investigadores, estudiantes e invitados prestigiosos en sus congresos, la publicación de casi una veintena de libros y cientos de artículos científicos, la impartición de cientos de cursos de posgrado, programas de maestría y doctorado, los 115 convenios de colaboración, la creación de una revista científica para la socialización de los resultados de alto impacto, así como la estructuración de una plataforma para el trabajo coordinado entre sus más de cinco mil miembros, demuestran la efectividad de la gestión que lleva a cabo la red y el meritorio liderazgo alcanzado en la región, lo cual tributa a las buenas prácticas en la promoción del desarrollo sustentable.

PALABRAS CLAVE: desarrollo endógeno, líderes comunitarios, sistematización, sustentabilidad

\section{ABSTRACT}

The objective of this work was to systematize the experiences of the Iberoamerican Environment Network (REIMA, AC) in the environmental training of community leaders for the management of endogenous development projects, with the active participation of vulnerable groups and young people in America Latin and the Caribbean. The investigation showed that REIMA, A.C. contributed to these purposes by facilitating the exchange of experiences, the carrying out of research and development projects, and the organization of academic events related to sustainability in Latin America; this as a result of the articulation between the more than 100 institutions that are part of it in 17 countries of the world. The wide participation of prestigious professors, researchers, students and guests in its congresses, the publication of almost twenty books and hundreds of scientific articles, the delivery of hundreds of postgraduate courses, master's and doctoral programs, the 115 collaboration agreements, the creation of a scientific journal for the socialization of high-impact results, 
as well as the structuring of a platform for coordinated work among its more than 5000 members, demonstrate the effectiveness of the management carried out by the network and the Meritorious leadership achieved in the region, which contributes to good practices in promoting sustainable development.

KEYWORDS: community leaders, endogenous development, sustainability, systematization

\section{RESUMO}

Um dos objetivos principais da Rede Ibero-americana de Maio Ambiente (REIMA-AC) é contribuir na formação ambiental de lideranças comunitárias para a gestão de projetos de desenvolvimento endógenos, promovendo a participação de grupos vulneráveis e da população jovem em geral. 0 presente trabalho foi sistematizar as experiências da REIMA-AC no campo da formação ambiental na América Latina e o Caribe. A REIMA-AC tem contribuído para a formação ambiental de milhares de docentes, pesquisadores, estudantes e outros atores sociais através do intercâmbio de experiências, a realização de projetos de pesquisa e desenvolvimento, bem como, a organização de eventos acadêmicos (Cursos, Seminários, Congressos, Oficinas, Encontros, Palestras e outros) relacionados com a sustentabilidade ambiental em Ibero-América. A articulação entre mais de 100 instituições que fazem parte da REIMA-AC em 17 países da América Latina, Caribe, Europa e América do Norte tem permitido a realização de dez congressos, a publicação de vinte livros e de centenas de artigos científicos, bem como, de centenas de cursos de pós-graduação; programas de mestrado e doutorado; todo isto a partir da estruturação de uma plataforma de trabalho coordenado entre os mais de cinco mil membros da rede.

PALAVRAS CHAVE: desenvolvimento endógeno, lideranças comunitárias, sistematização, sustentabilidade

\section{INTRODUCCIÓN}

La Conferencia de las Naciones Unidas sobre Medio Ambiente y Desarrollo, también conocida como "Cumbre de la Tierra», (Organización de Naciones Unidas [ONU], 1992) impulsó nuevas corrientes de pensamiento en torno a los efectos que, hasta esa fecha, la creciente industrialización de la sociedad había provocado sobre la naturaleza. Las posturas ecologistas manifestadas en los inicios de la segunda mitad del siglo XX, y luego favorecidas con las renovaciones conceptuales y prácticas que supuestamente - se llevarían a cabo tras la presentación del llamado Informe Brundtland (ONU, 1987); encontrarían en las entonces emergentes Tecnologías de la Información y la Comunicación (TIC), herramientas de trabajo estratégicas para sensibilizar a la humanidad sobre el necesario cambio en sus modos de actuación, como única vía posible para garantizar la preservación de la especie humana y todas las formas de vida y recursos en el Planeta.

Dentro de este contexto, marcado además por fuertes transformaciones en los planos geopolítico, industrial y cultural, etc., el avance de las telecomunicaciones propicia la consolidación de las redes de cooperación internacional para potenciar el desarrollo científico-tecnológico y la internacionalización de instituciones educativas, centros de investigación, empresas y organizaciones gubernamentales y no gubernamentales (ONG) en varias áreas del conocimiento; convirtiéndose las mismas no solo en instrumentos caracterizados por su versatilidad y eficacia, sino también en modelos organizativos para el fortalecimiento institucional y la articulación de sistemas de innovación, como señalara Sebastián (2000). 
En este sentido, a decir el autor, pueden definirse las redes de cooperación como «asociaciones de interesados que tienen como objetivo la consecución de resultados acordados conjuntamente (...) sobre la base de (...) la complementación de sus capacidades y la sinergia de sus interrelaciones (...) alrededor de un plan de acción» (Sebastián, 2000, p. 98).

Bajo esos preceptos, surgen por todo el orbe iniciativas caracterizadas por el abordaje multidisciplinario de temas novedosos para la época en las más disímiles formas de colaboración, en espacios físicos y virtuales. Tal es el caso de la Red Estudiantil Iberoamericana de Medio Ambiente, fundada en 1999 en la Universidad de La Habana e integrada por estudiantes universitarios de México, Cuba, Venezuela, Colombia y Brasil, la cual sería registrada como asociación civil sin fines de lucro en el estado de San Luis Potosí, México, en el 2004; y diez años después renovaría su proyecto bajo la denominación de Red Iberoamericana de Medio Ambiente (REIMA, A.C.), trasladándose posteriormente su coordinación general hacia Ecuador el 20 de marzo de 2015.

Experiencias similares lo constituyen la Red de Formación Ambiental para América Latina y el Caribe, del Programa de las Naciones Unidas para el Medio Ambiente; la Red de Autoridades Ambientales (RAA) de la Comisión Europea; la Alianza de Redes Iberoamericanas de Universidades por la Sustentabilidad y el Ambiente (ARIUSA); la Red de Medio Ambiente (RedMA), del Ministerio de Educación Superior de Cuba; la Red de Medio Ambiente (REMA-IPN), del Instituto Politécnico Nacional de México, por citar solo algunos ejemplos; las cuales tienen como fines comunes la promoción del cuidado y protección del ambiente desde los procesos de investigación, innovación y desarrollo, que respaldan la toma de decisiones y promueven el desarrollo sustentable demandado en varias asambleas y programas de la ONU, tales como el Programa 21 (ONU, 1992), la Declaración del Milenio (ONU, 2000), y más recientemente, la Agenda 2030 (ONU, 2015).

En noviembre de 2019 la REIMA, A.C. celebró su vigésimo aniversario con resultados reconocidos internacionalmente y que nutren el trabajo que se presenta, cuyo propósito fue sistematizar las experiencias de la Red Iberoamericana de Medio Ambiente en la formación ambiental de líderes comunitarios para la gestión de proyectos de desarrollo endógeno, con la participación activa de grupos vulnerables y población joven en América Latina y el Caribe. Los mismos son la expresión del quehacer mancomunado entre sus miembros para dar cumplimiento a los objetivos planteados como asociación civil:

Contribuir a la formación ambiental y al desarrollo sustentable en Iberoamérica, apegado a la política ambiental de cada país y a las estrategias gubernamentales, mediante:

a) El intercambio de experiencias a través de la realización de proyectos de investigación científica y aplicada; y eventos académicos relacionados con la gestión ambiental y el desarrollo sustentable.

b) La cooperación individual y colectiva entre instituciones públicas, privadas y demás organizaciones civiles a través de la participación en proyectos de gestión ambiental como contribución al desarrollo sustentable.

c) Alianzas estratégicas público-privadas que permitan la implementación de programas de educación formal y no formal que fortalezcan la gestión ambiental comunitaria.

d) La promoción de buenas prácticas ambientales a través de programas de voluntariado, conforme a la legislación de cada país. (REIMA, A.C., 2019a, p. 2) 
Los autores del presente artículo quieren agradecer a todos los coordinadores actuales de los Puntos Focales Nacionales de la red y a los que se han desempeñado en ese rol a lo largo de su historia, sin cuyo apoyo no hubiera sido posible fortalecer las relaciones de cooperación basadas en el respeto mutuo y el compromiso institucional como garantía del éxito y la calidad de todas las actividades emprendidas en su nombre; así como a todos los miembros de su Consejo Científico en igual condición, sin los cuales no pudiera realizarse el trabajo riguroso y personalizado en la gestión académica, científica y extensionista promovida por la REIMA, A.C. en Iberoamérica.

\section{MATERIALES Y MÉTODOS}

El estudio que se presenta tiene un marcado enfoque cualitativo al utilizar como principal método para su realización la sistematización de experiencias, cuyo fundamento tiene un respaldo epistemológico dialéctico - interpretativo (Ruiz, 2017). Para llevar a cabo el mismo se tuvieron en cuenta los criterios de Jara (1994, 2012), Freire (1996), Ruiz (2001), Speranza (2016) y Ruiz (2017), quienes de manera general lo reconocen como un proceso basado en el reconocimiento de la práctica, participativo y dialógico, donde resultan medulares tanto los resultados a los cuales se arriben, como el camino transitado para la obtención de estos.

Los autores de esta investigación asumen los criterios de Jara (2012) cuando asevera que es un "proceso de reflexión e interpretación crítica sobre la práctica y desde la práctica, que se realiza con base en la construcción y ordenamiento de los factores objetivos y subjetivos que han intervenido en la experiencia, para extraer aprendizajes y compartirlos» (p.67).

Siguiendo las etapas planteadas por el referido autor, fueron empleados además, como métodos teóricos fundamentales el histórico-lógico, con el fin de conocer los antecedentes y condiciones históricas que originaron la creación de la REIMA, A.C.; la inducción-deducción, para comparar las características de esta con definiciones validadas en la literatura sobre el tema; el análisis y síntesis, para examinar y procesar la información obtenida de los documentos consultados; y el sistémico-estructural, con el objetivo de abordar las características propias de la red. Como método empírico se utilizó el análisis documental para la revisión de los documentos asociados a su gestión, tales como actas, relatorías, ponencias, publicaciones periódicas, redes sociales, convenios, programas formativos (cursos, maestrías, doctorados), etc.

La sistematización de experiencias permite la generación de conocimientos a partir de la reflexión crítica de la praxis cotidiana, por lo cual al asumirlo como principio compositivo del estudio, se ha querido extraer toda la información necesaria para evaluar el impacto alcanzado por la REIMA, A.C. en su misión de «Promover activamente la concienciación sobre los principales problemas ambientales a escala local, nacional e internacional, buscando soluciones que ayuden a mitigar los impactos ambientales de la actividad humana que, de forma práctica, sean socialmente justas y económicamente viables» (REIMA, A.C., 2019a, p.1); para de esta manera perfeccionar su sistema de trabajo en virtud de reajustar sus líneas investigativas, según las prioridades de los contextos regionales y fortalecer las relaciones de cooperación entre sus asociados, con el fin de lograr los resultados que permitan validar la visión que como asociación tiene sobre sí misma: "Ser reconocida como una organización líder para la articulación social en función de la sustentabilidad ambiental en Iberoamérica a partir del principio de soluciones locales a problemas globales» (REIMA, A.C., 2019a, pp.1-2).

\section{RESULTADOS Y DISCUSIÓN}

El uso sustentable de la biodiversidad y manejo de áreas protegidas; el manejo sustentable de tierras y seguridad alimentaria; la gestión de riesgos ambientales y cambio climático; la gestión sustentable de recursos hídricos; la gestión ambiental en asentamientos humanos; la gestión sustentable de residuos sólidos urbanos; educación, cultura y comunicación ambientales; el turismo sustentable; la 
política y el derecho ambiental; las bases de datos; la percepción remota y SIG aplicados a la gestión ambiental; y la cooperación universitaria para el desarrollo sustentable; son los ejes temáticos que rigen la labor emprendida por la REIMA, A.C. en pos de la formación ambiental de sus más de cinco mil miembros.

Durante 20 años REIMA, A.C. ha logrado establecer relaciones de colaboración mediante convenios, programas y proyectos de investigación y desarrollo, que le han permitido diseñar sus actividades, acciones y tareas, atendiendo a los propios procesos educativos que promueve. En la actualidad integran la red 100 instituciones de reconocido prestigio internacional de 17 países de América Latina, el Caribe, Europa y Norteamérica (figura 1).

Figura 1. Instituciones aliadas a la REIMA, A.C. por países.

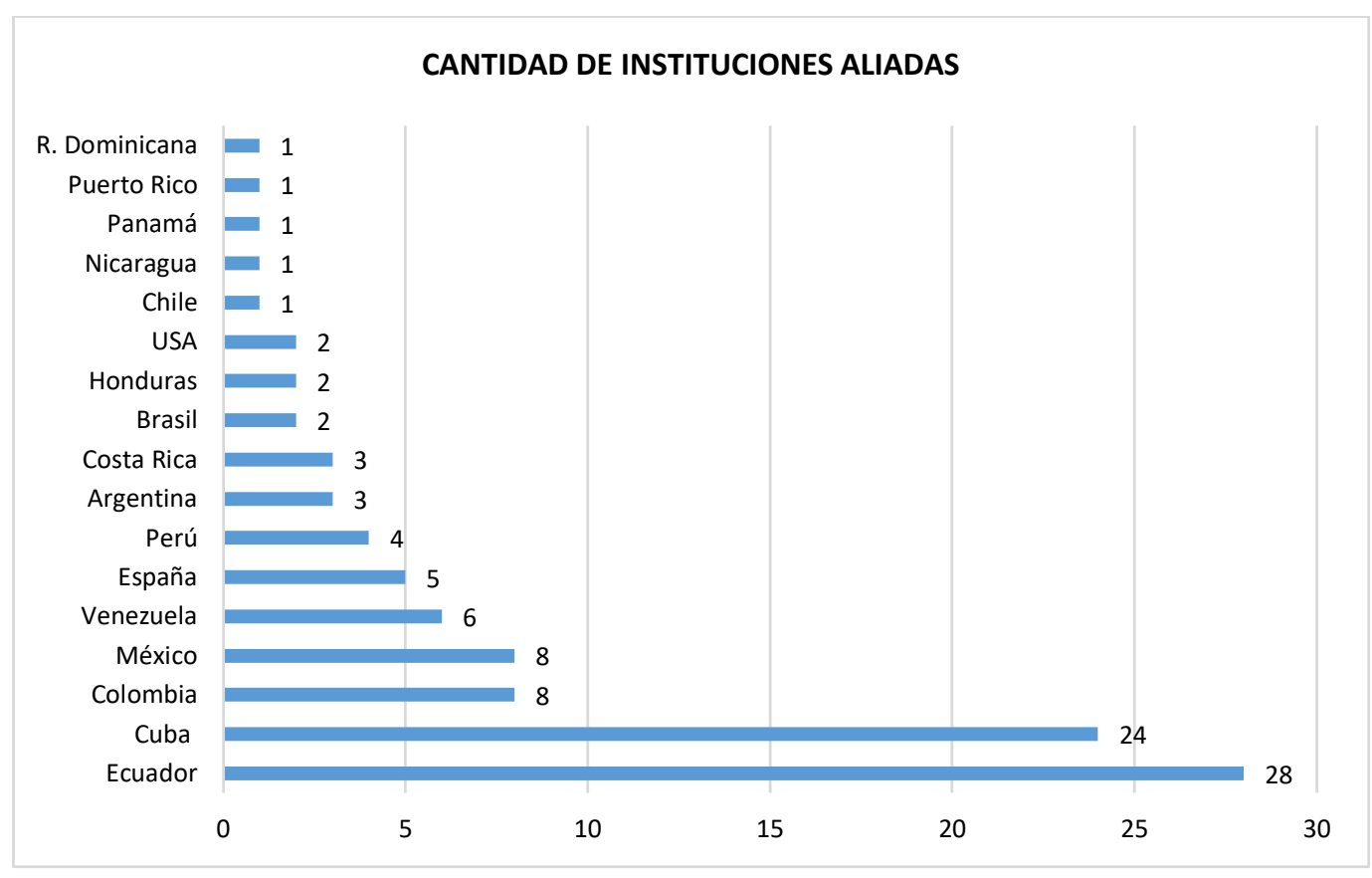

Fuente: REIMA, A.C. (2020a).

La labor como red académica y temática, de acuerdo con la clasificación ofrecida por Sebastián (2000), ha sido avalada oficialmente (REG-RED-18-0057) ' por la Secretaría de Educación Superior, Ciencia, Tecnología e Innovación (SENESCYT, 2020) y por el Misterio del Ambiente de la República de Ecuador; así como por los ministerios de Ciencia, Tecnología y Medio Ambiente y de Educación Superior, de la República de Cuba.

Asimismo, el alcance y carácter de su accionar, marcado por su compromiso y responsabilidad social en el fomento del desarrollo sustentable, le ha permitido ganarse el reconocimiento del Programa de las Naciones Unidas para el Medio Ambiente (UNEP, por sus siglas en inglés); del Programa de las Naciones Unidas para el Desarrollo (UNDP, por sus siglas en inglés) y del Programa de Pequeñas Donaciones del Fondo Mundial para el Medio Ambiente (SGP/GEF, por sus siglas en inglés). Lo anterior supone un fuerte trabajo docente, investigativo y de vinculación social, como se reflejará a continuación.

\footnotetext{
${ }^{1}$ https://reima-ec.org/reconocimiento-senescyt
} 


\section{Formación ambiental para la sustentabilidad.}

Durante el período 2015-2019, la REIMA, A.C. ofreció, en alianza con sus socios estratégicos, un total de 187 programas de posgrado que incluyeron cursos de superación profesional, diplomados, especialidades, maestrías y doctorados; los cuales permitieron la formación de más de 3000 estudiantes, tal como se expresa en las figuras 2 y 3 que aparecen a continuación:

Figura 2. Posgrados impartidos como parte del Programa de Formación Ambiental.

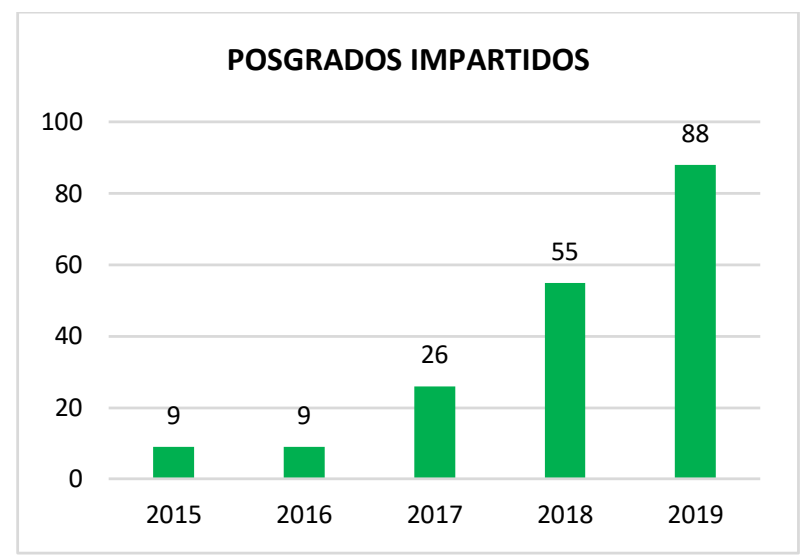

Figura 3. Egresados del Programa de Formación Ambiental.

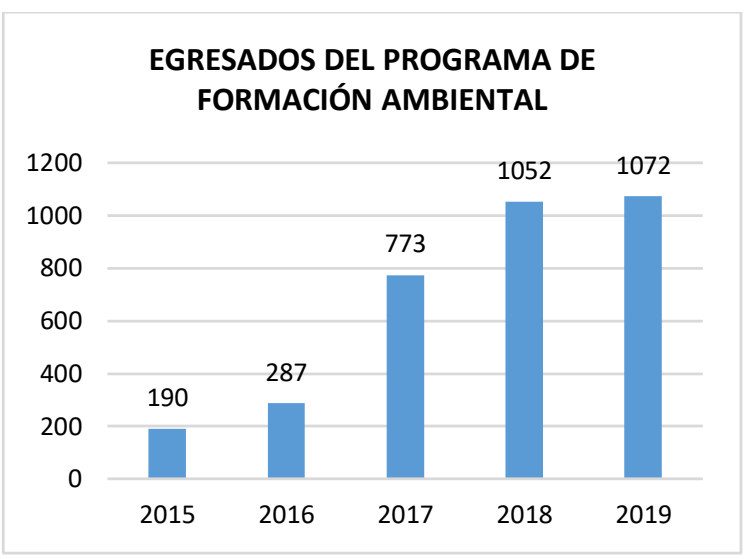

Fuente: Puerta, Ulloa y Martínez (2020).

Dentro de esta oferta académica destacan las alianzas con Fondo Verde Internacional de Perú y con la Universidad Centro Panamericano de Estudios Superiores de México, sobre todo en lo relacionado con los siguientes programas (Álvarez y Puerta, 2019):

Doctorados en Sostenibilidad

- Doctorado en Conservación y Restauración del Medio Natural

- Doctorado en Proyectos: Línea de Investigación en Medio Ambiente

- Doctorado en Investigación y Docencia

El Fondo Verde Internacional, de conjunto con la Universidad de Salamanca, España, ofrece el MBA en Turismo Sostenible y el Máster en Gestión Sostenible del Ambiente. Por su parte, la Universidad Centro Panamericano de Estudios Superiores propicia los siguientes programas de maestría:

- Maestría en Gestión y Auditorías Ambientales

- Maestría en Ingeniería y Tecnología Ambiental

- Maestría en Ciencia y Tecnología Marina

- Maestría en Gestión Integrada de Prevención, Medio Ambiente y Calidad 
Acompañamiento e intercambio de experiencias sobre gestión ambiental.

De igual manera, cumpliendo con el objetivo de facilitar el intercambio de experiencias a través de la realización de proyectos de investigación científica y aplicada, y eventos académicos relacionados con la gestión ambiental y el desarrollo sustentable, la REIMA, A.C. ha logrado posicionar varios eventos ${ }^{2}$ dentro del ranking de su tipo en América Latina y el Caribe, son estos:

- Congreso Iberoamericano sobre Educación Ambiental para la Sustentabilidad

- Congreso Iberoamericano sobre Manejo Sustentable de Tierras y Seguridad Alimentaria

- Congreso Iberoamericano sobre Ambiente y Sustentabilidad

- Congreso Iberoamericano sobre Turismo Sustentable

- Seminario Científico Internacional sobre Cooperación Universitaria para el Desarrollo Sustentable

- Taller Estudiantil Internacional sobre Medio Ambiente (TEIMA)

En este sentido, los congresos organizados en Cuba, Colombia, Ecuador y Honduras, han contado con la participación de más de 4900 delegados e invitados de 26 países, en los últimos cinco años. Se reflejan dichos datos en las figuras 4 y 5 :

Figura 4. Cantidad de participantes en eventos seleccionados organizados por REIMA, A.C.

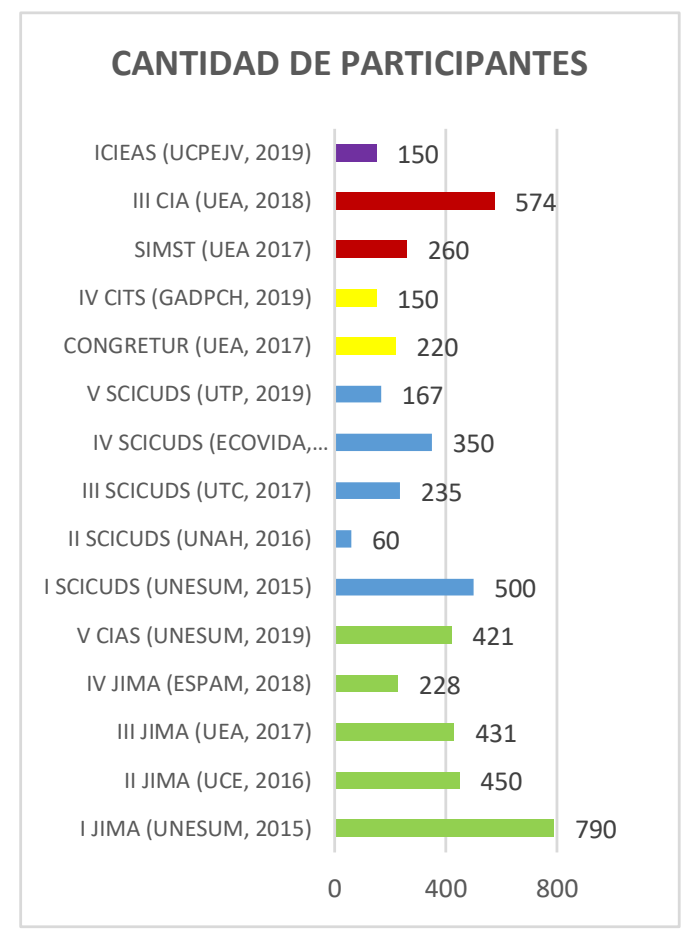

Figura 5. Participantes extranjeros en eventos organizados por REIMA, A.C.

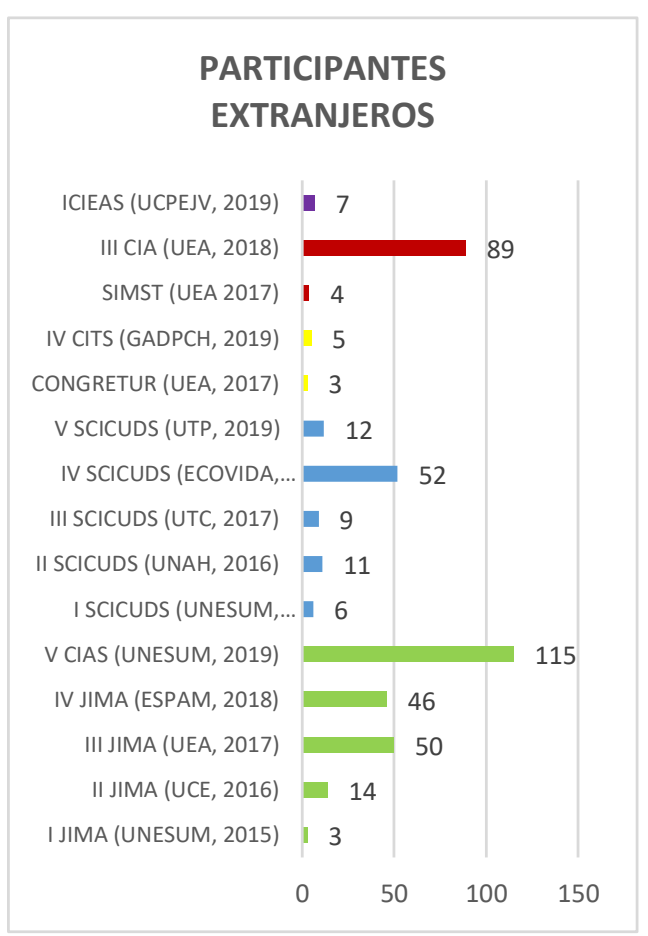

Fuente: Puerta, Ulloa y Martínez (2020).

\footnotetext{
2 https://reima-ec.org/memoria-de-eventos/
} 
Los países con mayor representación son Angola, Argentina, Belice, Bolivia, Brasil, Canadá, Colombia, Costa Rica, Cuba, Chile, Ecuador, El Salvador, España, Estados Unidos de América, Honduras, Guatemala, Italia, México, Nicaragua, Panamá, Perú, Puerto Rico, República Dominicana, Uruguay y Venezuela.

Asimismo, la presencia de más de 500 instituciones mediante prestigiosos docentes, investigadores, decisores y estudiantes en estos, según las relatorías de los mismos, demuestran que, en efecto, la REIMA, A.C. se ha convertido en un espacio para el intercambio de experiencias y la articulación social a favor de la sustentabilidad ambiental en América Latina y el Caribe.

\section{Comunicación ambiental para la sustentabilidad.}

No solo son los eventos un indicador de la visibilidad del quehacer cotidiano de la red; la publicación de ponencias y artículos científicos a través de diferentes productos destinados a la comunicación científica en virtud de lograr el acceso de un público heterogéneo a la información recogida en ellos, constituye una de las contribuciones de mayor impacto.

En total, de 1723 trabajos propuestos para su publicación en alguna de las modalidades establecidas por su Sello Editorial Ambiente \& Sustentabilidad, han sido aprobados 477, lo que representa el $27.68 \%$ de los mismos. Este valor es el resultado de un riguroso proceso de revisión por pares, lo mismo para su colección Memorias..., con sus respectivos números de ISBN (REIMA, A.C., 2020b), como para su Revista Iberoamericana Ambiente \& Sustentabilidad ${ }^{3}$, publicación principal que cuenta con su respectivo número de ISSN y registro SENESCYT, destinada a socializar los resultados de alto impacto (figuras 6 y 7).

Figura 6. Ponencias presentadas en eventos organizados por REIMA, A.C.

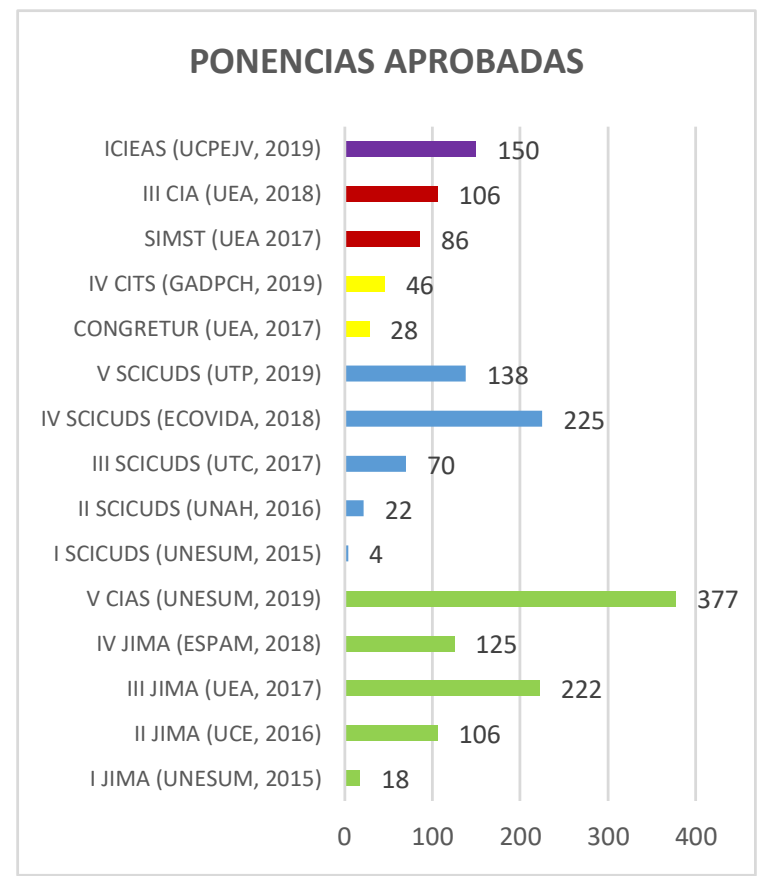

Figura 7. Artículos científicos publicados por REIMA, A.C.

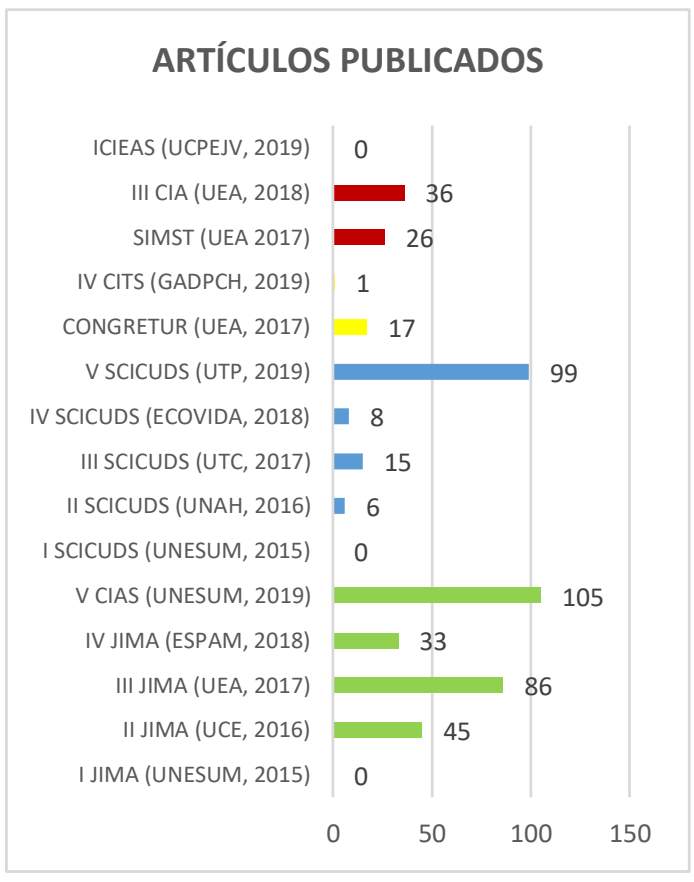

Fuente: Puerta, Ulloa y Martínez (2020).

\footnotetext{
${ }^{3}$ https://ambiente-sustentabilidad.org/index.php/revista
} 
Importante ha sido el esfuerzo de los Nodos y Puntos Focales Nacionales para dar a conocer las obras que se relacionan a continuación (Valero, Cisneros y Rodríguez, 2019):

- Memorias Il Jornada Iberoamericana en Saludo al Día Mundial del Medio Ambiente (Ecuador, 2016). ISBN: 978-9942-14-459-1

- Memorias II Seminario Científico Internacional de Cooperación Universitaria para el Desarrollo Sostenible (Honduras, 2016. ISSN 2225-5249) y Revista Ciencias Espaciales Vol. 10, No. 2, otoño 2017. ISSN 2521- 5868

- Recursos Naturais e Convivência Sustentável no Semiárido (Brasil, 2017). ISBN: 978-85-9205495-3

- Memorias III Jornada Iberoamericana en Saludo al Día Mundial del Medio Ambiente (Ecuador, 2017). ISBN: 978-9942-932-20-4

- Memorias Científicas del III CONGRETUR "Hacia la Sostenibilidad Turística» (Ecuador, 2017). ISBN: 978-9942-932-25-9

- Memorias Simposio Internacional sobre Manejo Sostenible de Tierras y Seguridad Alimentaria (Ecuador, 2017). ISBN: 978-9942-932-22-8

- Memorias del IV Workshop Nacional de Meio Ambiente e Sustentabilidade nos territórios Semiáridos. Desafios Socioambientais e Proteção do Meio Ambiente. (Brasil, 2018). ISBN: 97885-920549-6-0

- Memorias II Encuentro Científico Nacional de Educación Ambiental y Desarrollo Sostenible (Cuba, 2018). ISBN 978-959-16-3918-9

- Ambiente y Sustentabilidad Volumen 5. V Congreso Iberoamericano sobre Ambiente y Sustentabilidad. Libro de resúmenes (Ecuador, 2019). ISBN: 978-9942-36-201-8

- Ambiente y Sustentabilidad Volumen 6. IV Congreso Iberoamericano sobre Turismo Sustentable. Libro de resúmenes (Ecuador, 2019). ISBN: 978-9942-36-583-5

Aun cuando posee su propio sello editorial, la REIMA, A.C. colabora, además, con los comités editoriales de siguientes revistas (Valero, Cisneros y Rodríguez, 2019):

- Revista Cubana de Ciencias Forestales (UPR, Cuba). ISSN: 2310-3469

- Revista La Técnica (UTM, Ecuador). ISSN: 1390-6895; e-ISSN: 2477-8982

- Revista ECOVIDA (ECOVIDA, Cuba). ISSN: 2076-281X

\section{Cooperación para el desarrollo.}

Establecer alianzas estratégicas público-privadas, que permitan la implementación de programas de educación formal y no formal para el fortalecimiento de la gestión ambiental comunitaria, constituye otro de los objetivos de la REIMA, A.C.; es por ello que durante el período 2015-2019 se han firmado un total de 115 Acuerdos/Convenios de cooperación ${ }^{4}$ (figura 8).

\footnotetext{
${ }^{4}$ https://reima-ec.org/convenios/
} 
Figura 8. Acuerdos/Convenios de cooperación suscritos por países.

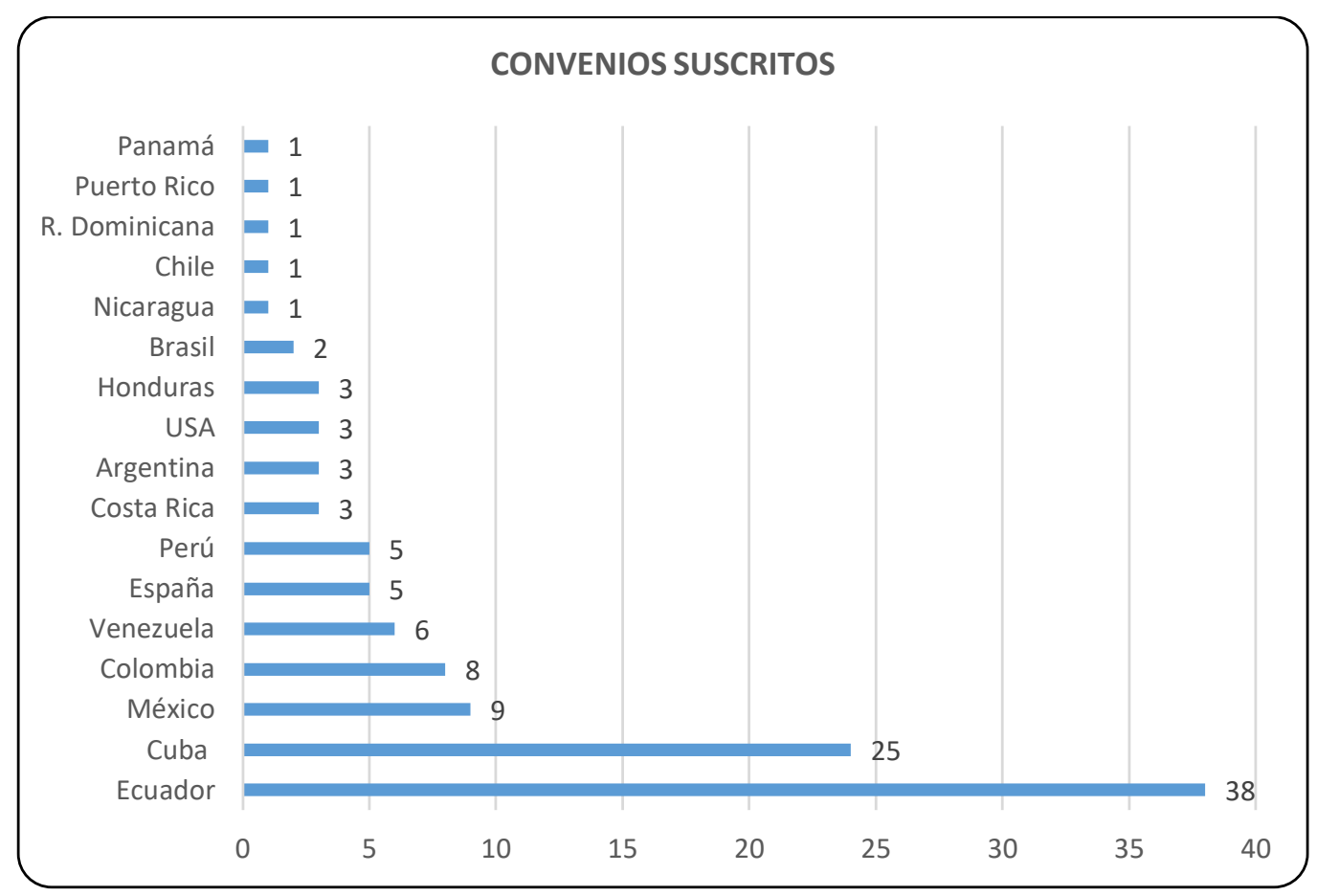

Fuente: REIMA, A.C. (2020b)

Gracias a dichas alianzas se han podido promover las buenas prácticas ambientales a través de programas de voluntariado, conforme a la legislación de cada país. De igual manera, durante el período 2015-2019 se han realizado, según el cronograma establecido, las Asambleas Generales Anuales de la asociación:

- Junio 2015. Universidad Estatal del Sur de Manabí (UNESUM), Ecuador.

- Junio 2016. Universidad Central del Ecuador (UCE), Ecuador.

- Junio 2017. Universidad Estatal Amazónica (UEA), Ecuador.

- Junio 2018. Escuela Superior Politécnica Agropecuaria de Manabí (ESPAM), Ecuador.

- Noviembre 2018. Centro de Investigaciones y Servicios Ambientales (ECOVIDA), Cuba.

- Junio 2019. Universidad Estatal del Sur de Manabí (UNESUM), Ecuador.

- Noviembre 2019. Universidad Tecnológica de Pereira (UTP), Colombia.

Dentro de los logros de estos espacios, destinados al análisis del funcionamiento interno y el cumplimiento del marco legal establecido para la cooperación interinstitucional, resultan imprescindibles mencionar los acuerdos que propiciaron:

- La creación del Premio Iberoamericano de Medio Ambiente Dr. José Manuel Mateo Rodríguez, en reconocimiento al extraordinario aporte de este científico latinoamericano al pensamiento ambiental y construcción teórica de la sustentabilidad; el cual es entregado anualmente a instituciones, proyectos comunitarios y personas naturales que se destacan por su contribución a la gestión ambiental y la sustentabilidad en cualquiera de los países del espacio iberoamericano. Su otorgamiento es aprobado por la Asamblea General de la REIMA, A.C. a propuesta de los Puntos Focales Nacionales. 
- La entrega de la Placa XX Aniversario de la Red Iberoamericana de Medio Ambiente a 20 instituciones cuya contribución al trabajo de la misma durante estos 20 años ha sido relevante. De tal distinción fueron merecedores:

1. Universidad Agraria de La Habana Fructuoso Rodríguez, Cuba

2. Universidad Tecnológica de La Habana José Antonio Echeverría, Cuba

3. Universidad de La Habana, Cuba

4. Centro de Investigaciones y Servicios Ambientales de Pinar del Río ECOVIDA, Cuba

5. Universidad de Ciencias Pedagógicas Enrique José Varona, Cuba

6. Agencia de Medio Ambiente, Cuba

7. Programa de Asociación de País sobre Manejo Sostenible de Tierras (CPP-OP15), Cuba

8. Universidad Estatal del Sur de Manabí, Ecuador

9. Universidad Central del Ecuador, Ecuador

10. Universidad Estatal Amazónica, Ecuador

11. Universidad Metropolitana, Ecuador

12. Universidad Técnica de Cotopaxi, Ecuador

13. Escuela Superior Politécnica Agropecuaria de Manabí Manuel Félix López, Ecuador

14. Gobierno Autónomo Descentralizado de la provincia Chimborazo, Ecuador

15. Centro Ecuatoriano para la Gestión Ambiental y el Desarrollo Sostenible, Ecuador

16. Agua purificada, envasada sin gas SULTANA, Ecuador

17. Universidad Nacional Autónoma de Honduras, Honduras

18. Universidad Tecnológica de Pereira, Colombia

19. Universidad Centro Panamericano de Estudios Superiores, México

20. Fondo Verde, Perú

En la Asamblea General Anual de la REIMA, A.C. (2019-II), se reconoció el aporte económico durante el período 2015-2019, al haber otorgado más de 900 becas que hicieron posible que docentes, investigadores y estudiantes latinoamericanos pudieran participar en los principales eventos organizados por la red (congresos, seminarios, talleres, cursos de posgrado, etcétera), con una inversión de más de 156 mil USD en boletos de avión, viáticos, transporte terrestre, cuota de inscripción y otras ayudas económicas (REIMA, A.C., 2019b).

Grosso modo, los resultados hasta aquí expuestos validan lo planteado por Sebastián (2000), quien afirma que:

La cooperación supone siempre abrir espacios para compartirlos, debiendo asumirse con tolerancia las diferencias culturales y procurando aprovechar estas diferencias para ampliar el ámbito de las experiencias personales e institucionales. Las redes suelen crear ambientes propicios para la confraternización y el conocimiento mutuo, que figuran entre sus mejores resultados intangibles (p. 106). 


\section{CONCLUSIONES}

El proceso de sistematización de experiencias demostró que la REIMA, A.C. ha contribuido significativamente a la formación ambiental de líderes comunitarios para la gestión de proyectos de desarrollo endógeno, con la participación de grupos vulnerables y población joven en general, con un amplio alcance y factibilidad en su gestión; cumpliéndose con creces desde el punto de vista cualitativo y cuantitativo los objetivos que se ha planteado como asociación civil. Ello ha traído consigo la realización de proyectos de investigación y desarrollo, la organización de eventos académicos, la publicación de resultados científicos de alto impacto y el establecimiento de más de 100 convenios de colaboración, con el fin de orientar la cultura ambiental de Iberoamérica hacia los principios de la sustentabilidad.

\section{REFERENCIAS BIBLIOGRÁFICAS}

Álvarez, Y., y Puerta, Y. (2019, 13 de abril). Los Objetivos de Desarrollo Sostenible: una oportunidad para la acción. [Ponencia]. Convención Internacional Varona 2019, La Habana, Cuba.

Freire, P. (1996). Política y Educación. Editorial Siglo XXI.

Jara, O. (1994). Para sistematizar experiencias: una propuesta teórica y práctica (1 ${ }^{a}$ ed.). ALFORJA. https://url2.cl/FBxl2

Jara, O. (2012). Sistematización de experiencias, investigación y evaluación: aproximaciones desde tres ángulos. Revista Internacional sobre Investigación en Educación Global y para el Desarrollo, (1), 56-70. https://url2.cl/aPxLf

Organización de Naciones Unidas. (1987). Nuestro futuro común. Comisión Mundial sobre Medio Ambiente y Desarrollo. https://url2.cl/t2q5Z

Organización de Naciones Unidas. (1992). Programa 21. https://url2.cl/misv5

Organización de Naciones Unidas. (2000). Declaración del Milenio. https://url2.cl/3KitV

Organización de Naciones Unidas. (2015). Transformar nuestro mundo: Agenda 2030 para el Desarrollo Sostenible. https://url2.cl/InqP1

Puerta, Y., Ulloa, S., y Martínez, A. (2019, 15 de noviembre). Contribución de la Red Iberoamericana de Medio Ambiente a la formación ambiental en América Latina y el Caribe. [Ponencia]. V Seminario Científico Internacional de Cooperación Universitaria para el Desarrollo Sostenible, Pereira, Colombia.

Puerta, Y., Ulloa, S., y Martínez, A. (2020, 16 de febrero). Contribución de la Red Iberoamericana de Medio Ambiente a la formación ambiental en América Latina y el Caribe [Ponencia]. II Congreso Iberoamericano sobre Educación Ambiental para la Sustentabilidad, Riobamba, Ecuador.

Red Iberoamericana de Medio Ambiente, A.C. (2019a). Estatuto. https://reimaec.org/documentos constitutivos/

Red Iberoamericana de Medio Ambiente, A.C. (2019b). Acta de la Asamblea General Anual 2019-II. https://reima-ec.org/informes-de-gestion/

Red Iberoamericana de Medio Ambiente, A.C. (2020a). Alianzas estratégicas. https://reimaec.org/alianzas-estrategicas/ 
Red Iberoamericana de Medio Ambiente, A.C. (2020b). Informe de Gestión 2015-2019. https://reimaec.org/informes-de-gestion/

Ruiz, L.D. (2001). La sistematización de prácticas. https://url2.cl/6AKts

Ruiz, W. (2017). Sistematización de experiencias: La práctica educativa de un maestro de filosofía [Tesis de maestría, Universidad de San Buenaventura]. Repositorio institucional. https://url2.cl/uTtDh

Sebastián, J. (2000). Las Redes de Cooperación como modelo organizativo y funcional para la I+D. REDES, 7(15), 97-111. http://ridaa.unq.edu.ar/handle/20.500.11807/702

Secretaría de Educación Superior, Ciencia, Tecnología e Innovación. (2020). Oficio Nro. SENESCYT -SGCTSDIC-2019-0060-CO. Registro de la red académica: Red Iberoamericana de Medio Ambiente (REIMA, A.C.). https://reima-ec.org/reconocimiento-senescyt/

Speranza, M. (2016). Sistematización de experiencias. Creando sentidos y aprendiendo de la práctica. Programa Federal de Apoyo al Desarrollo Rural Sustentable (ProFeder). https://url2.cl/mQh3U

Valero, D., Cisneros, Y., y Rodríguez, E. (2019, 6 de diciembre): Red Iberoamericana de Medio Ambiente (REIMA, A.C. - Cuba), nodo occidental. [Ponencia]. Simposio Internacional de Manejo Integrado y Gestión Ambiental de Playas y Ecosistemas Costeros, Varadero, Cuba. 